\title{
Sequestosome-1 (SQSTM1) sequence variants in ALS cases in the UK: prevalence and coexistence of SQSTM1 mutations in ALS kindred with PDB
}

\author{
Chun T Kwok ${ }^{1}$, Alex Morris ${ }^{1}$ and Jacqueline S de Belleroche ${ }^{\star 1}$
}

Mutations in the SQSTM1 gene have been reported to be associated with amyotrophic lateral sclerosis (ALS). We sought to determine the frequency of these mutations in a UK familial ALS (FALS) cohort. Sequences of all eight exons of the SQSTM1 gene were analysed in index cases from 61 different FALS kindred lacking known FALS mutations. Six exonic variants c.463G $>$ A, p.(Glu155Lys), c.822G $>$ C, p.(Glu274Asp), c.888G $>$ T, p. $(=)$, c.954C $>$ T, p. $(=)$, c.1038G $>$ A, p. $(=)$ and c.1175C $>$ T, p.(Pro392Leu) were identified in five FALS index cases, three of which were non-synonymous and three were synonymous. One index case harboured three variants (c.822G $>\mathrm{C}, \mathrm{c} .888 \mathrm{G}>\mathrm{T}$ and $\mathrm{c.954C}>\mathrm{T}$ ), and a second index case harboured two variants (c.822G $>\mathrm{C}$ and c.954C $>\mathrm{T}$ ). Only the p.(Pro392Leu) and p.(Glu155Lys) mutations were predicted to be pathogenic. In one p.(Pro392Leu) kindred, the carrier developed both ALS and Paget's disease of bone (PDB), and, in the p.(Glu155Lys) kindred, the father of the proband developed PDB. All p.(Pro392Leu) carriers were heterozygous for a previously reported founder haplotype for PDB, where this mutation has an established causal effect. The frequency of the p.(Pro392Leu) mutation in this UK FALS cohort was $2.3 \%$ and $0.97 \%$ overall including three previously screened FALS cohorts. Our results confirm the presence of the p.(Pro392Leu) SQSTM1 mutation in FALS. This mutation is the most common SQSTM1 mutation found in ALS to date, and a likely pathogenicity is supported by having an established causal role in PDB. The occurrence of the same mutation in ALS and PDB is indicative of a common pathogenic pathway that converges on protein homeostasis. European Journal of Human Genetics (2014) 22, 492-496; doi:10.1038/ejhg.2013.184; published online 14 August 2013

Keywords: p62; sequestosome-1(SQSTM1); amyotrophic lateral sclerosis; familial ALS; paget's disease of bone

\section{INTRODUCTION}

Considerable advances in understanding amyotrophic lateral sclerosis (ALS) pathogenesis have emerged from the identification of new mutations that cause familial ALS (FALS). ${ }^{1}$ These include TARDBP, FUS and C9ORF72 mutations that are associated with TDP43-positive poly-ubiquitinated inclusions, the hallmark of most cases of sporadic ALS. These inclusions not only contain TDP-43 or FUS but also p62/sequestosome 1 (p62/SQSTM1), optineurin $(O P T N)$ and ubiquilin-2 (UBQLN2). Indeed, mutations in several components of protein homeostasis, such as UBQLN2, charged multivesicular body protein $2 \mathrm{~B}(C H M P 2 B), O P T N$ and valosin containing protein $(V C P)$, are also present in less prevalent forms of ALS and ALS/FTD. ${ }^{2}$ Interestingly, mutations in OPTN and VCP are also seen in other allelic forms characterised by TDP-43-positive polyubiquitinated inclusions, primary open angle glaucoma (POAG), and inclusion body myopathy with Paget's disease of bone and frontotemporal dementia (IBMPFD), respectively.

Screening of four large ALS cohorts from United States, Europe and Japan for mutations in SQSTM1 has revealed the presence of a number of novel or rare coding mutations in FALS, FTLD and SALS, some of which are predicted to be pathogenic. ${ }^{3-6}$ However, because of lack of DNA in multiple members of FALS kindred, to date it has not been proved that any of these mutations are transmitted with disease in ALS. Nevertheless, among the coding mutations is p.(Pro392Leu), which is common in Paget's disease of bone (PDB), ${ }^{7,8}$ where there is evidence of transmission of the mutation with disease in multiple kindred. In order to further investigate and assess whether they are independently causal for ALS or whether p62/SQSTM1 DNA variants or modified forms of p62/SQSTM1 contribute to a significant predisposition to disease, we have examined a further FALS cohort from the United Kingdom.

p62/SQSTM1 is abundantly expressed in the spinal cord, especially in motor neurons and is present in distinctive p62/SQSTM1-positive fibrillar or compact TDP-43-positive inclusions found in ALS and FTD-ALS. ${ }^{9}$ In addition, a significant portion of C9ORF72-positive FTD/ALS patients have p62 positive, TDP-43-negative inclusions, indicating that multiple substrates are recognised by $\mathrm{p} 62$ in these conditions. ${ }^{10}$ SQSTM1 has multiple roles in protein homeostasis, ${ }^{11}$ in particular, the interaction of p62/SQSTM1 with the autophagic marker light chain 3 (LC3) is essential for the generation of autophagosomes necessary for the autophagic degradation of ubiquitinated protein aggregates. ${ }^{12}$ Depletion of p62 protein levels inhibits LC3 recruitment to autophagosomes and has been shown to increase cell death induced by mutant huntingtin. ${ }^{13}$

\section{PATIENTS AND METHODS}

Sample collection

The FALS cohort used in the initial screen of SQSTM1 mutations consisted of 61 FALS kindred that are known to lack mutations in SOD1, TARDBP, FUS, VAPB, DAO, VCP and C9ORF72. This is a subset of a larger cohort in which

${ }^{1}$ Neurogenetics Group, Division of Brain Sciences, Faculty of Medicine, Imperial College London, Hammersmith Hospital Campus, London, UK

*Correspondence: Professor JS de Belleroche, Neurogenetics Group, Division of Brain Sciences, Faculty of Medicine, Imperial College London, Hammersmith Hospital Campus, London W12 ONN, UK. Tel: + 44207 5946649; Fax: + 44207 59465448; E-mail: j.belleroche@imperial.ac.uk

Received 16 April 2013; revised 12 July 2013; accepted 19 July 2013; published online 14 August 2013 
known mutations have been previously characterised and has the following distribution of FALS mutations, C9ORF72 (30.2\%), SOD1 (18.0\%), TARDBP (4.3\%), FUS (2.2\%), DAO (0.72\%) and VAPB $(0.72 \%){ }^{14-19}$ This study employed ALS cases, presenting with motor neuron symptoms and diagnosed as ALS according to EL-Escorial criteria, from the United Kingdom (Imperial College Healthcare NHS trust). All ALS patients were positive for a familial history (FALS), and each patient was an index case from a separate kindred. Research governance regulations of the college were satisfied with appropriate informed consent from subjects. Controls included consented UK cases obtained within the Trust and genotype data from samples from the EUR subgroup of 1000 genome project (http:// www.1000genomes.org) and the European American subgroup of NHLBI EVS (http://evs.gs.washington.edu/EVS/). DNA was extracted from whole blood or the buffy coat layer using a DNA extraction kit (Qiagen, Manchester, UK). In addition, a further group of 26 C9ORF72-positive FALS cases were also screened.

\section{Genotyping}

All eight exons of the SQSTM1 gene that encode the major 440 amino-acid isoform (SQSTM1-001, NM_003900.4) were amplified using polymerase chain reaction (PCR). Primers flanking at least 50bp of these exons were designed using a Primer 3 program (http://frodo.wi.mit.edu/primer3) (Supplementary Information) and were purchased from Invitrogen (Paisley, UK). PCR products were purified using Sureclean (Bioline, London, UK), and sequenced using an ABI Prism BigDye terminator kit (Applied Biosystems, Warrington, Cheshire, UK). The presence of the p.(Pro392Leu) mutation was confirmed by RFLP analysis using the restriction enzyme Bst $\mathrm{U} 1$ (New England Biolabs, Hitchin, UK). DNA fragments were separated on a $2 \%$ agarose gel (Electran, VWR) and stained with $0.5 \mu \mathrm{g} / \mathrm{ml}$ ethidium bromide for $1 \mathrm{~h}$ at $11 \mathrm{~V} / \mathrm{cm} . \varphi \mathrm{X}-174-\mathrm{HaeIII}$ (New England Biolabs) was used as DNA marker. Gel profiles were visualized and analysed using a GelDoc system (Bio-Rad, Hertfordshire, UK). Novel variants have been submitted to DbSNP, (http://www.ncbi.nlm.nih.gov/SNP/) (JDB 2013 NCBI ss 0825679008-10).

\section{Data analysis}

Mutation pathogenicity was evaluated using SIFT (http://sift.jcvi.org), Polyphen 2 (http://genetics.bwh.harvard.edu/pph2/) and Mutation taster (http://www.mutationtaster.org). The effects of intronic variants on splicing were analysed using a Human splicing finder program (http://www.umd.be/ $\mathrm{HSF} /$ ). Genotype and allelic distributions of variants were compared with controls using $2 \times 2$ and $2 \times 3$ Fisher's tests and subjected to Benjamini-Hochberg correction. To examine genetic background, haplotypes were reconstructed using the Expectation-Maximization algorithm, implemented in PLINK. Linkage disequilibrium was measured by $r^{2}$ values calculated using Haploview (http://www.broadinstitute.org/haploview). We also analysed the age of onset and survival time of FALS using the Kaplan-Meier curve and log-rank test. The combined effect of pathogenic variants was calculated using the Mantel-Haenszel test.

\section{RESULTS}

Identification of SQSTM1 sequence variants in a UK-FALS cohort We screened all eight coding exons of the SQSTM1 gene (NM_003900.4) (Figure 1a) in a cohort of 61 Familial ALS patients. All subjects lacked mutations in SOD1, TARDBP, FUS (exons 14 and 15, which harbour most known mutations found in ALS), VAPB, $D A O, V C P$ and C9ORF72 genes, and each individual was an index case from unrelated families. Six exonic variants c.463G $>$ A, p.(Glu155Lys) (Figure 1b), c.822G $>$ C, p.(Glu274Asp), c.888G $>$ T, p. $(=)$, c. $954 \mathrm{C}>\mathrm{T}, \mathrm{p} .(=), \mathrm{c} .1038 \mathrm{G}>\mathrm{A}, \mathrm{p} .(=)$ and $\mathrm{c} .1175 \mathrm{C}>\mathrm{T}$, p.(Pro392Leu) (Figure 1c) were identified in five FALS index cases, three of which were non-synonymous and three were synonymous. One index case harboured three variants (c.822G $>C, c .888 \mathrm{G}>\mathrm{T}$ and c.954C $>\mathrm{T})$ and a second index case harboured two variants (c.822G $>$ C, c.954C $>$ T).

The c.822G > C (rs55793208, p.(Glu274Asp)) substitution has been previously reported in ALS by Rubino et al. ${ }^{4}$ and results in a
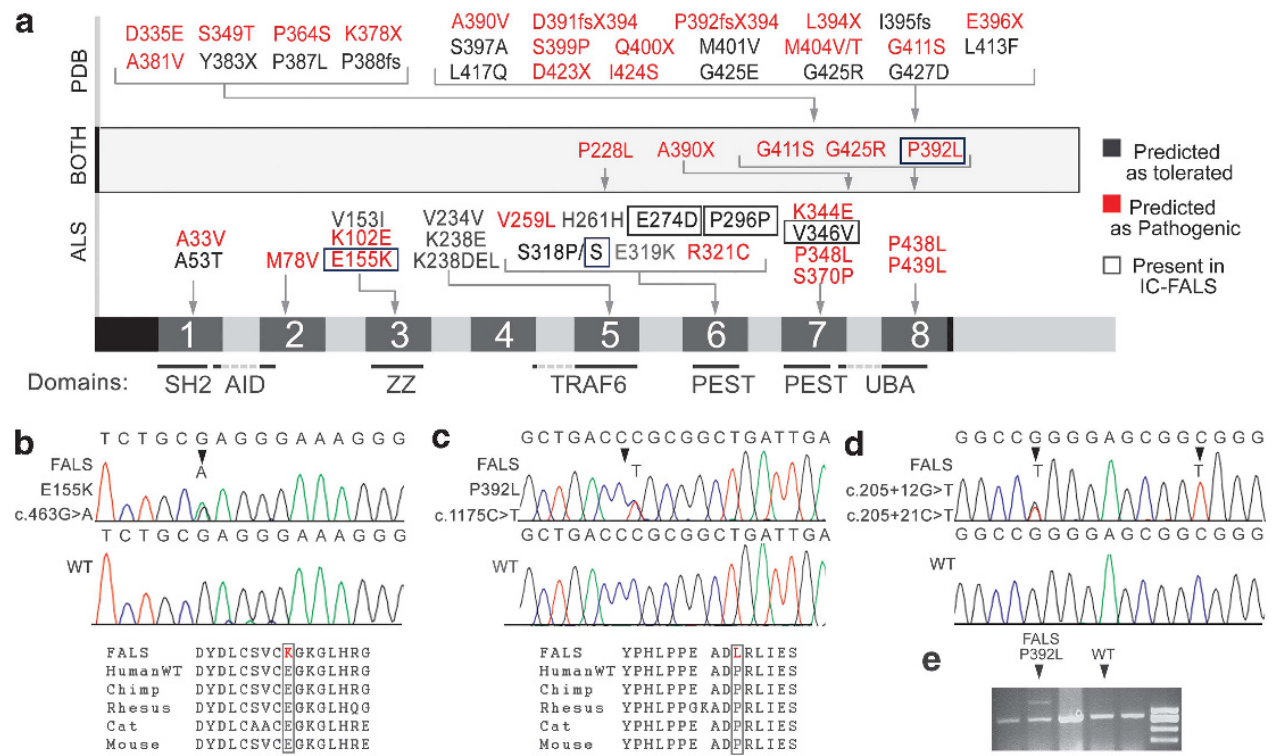

Figure 1 Sequence variants in SQSTM1 in ALS and PDB. (a) Summarizes SQSTM1 mutations previously reported in ALS and PDB. Probable pathogenic variants are in red and variants in the present study are outlined in blue. Domains are annotated as summarized by Geetha et al. ${ }^{11}$ SH2 (src homology 2 binding domain), AID(acidic interaction domain), ZZ (ZZ finger), TRAF6 (binding site for ring-finger protein tumour necrosis factor), PEST (PEST sequence) and UBA (Ubiquitin-associated domain). (b-d) Representative chromatographs showing the heterozygous c.463G $>$ A, p.(Glu155Lys), c.1175C $>T$, p.(Pro392Leu), c. $205+12 \mathrm{G}>\mathrm{T}, \quad$ c. $205+21 \mathrm{C}>\mathrm{T}$ variants and control sequences. The protein sequences are conserved in mammals. (e) The p.(Pro392Leu) mutation was confirmed by RFLP. Upper band showing the mutation only appeared in lane 2, whereas samples with normal sequences (other lanes) were completely digested. 
glutamate to aspartate change in the PEST domain of SQSTM1. All three synonymous variants have been previously reported, but not in ALS. The p.(Glu274Asp) and other synonymous variants were predicted to be tolerated, using SIFT, Polyphen2 and Mutation taster programs. However, while such programs can predict pathogenicity for recessive mutations, their predictions may not always apply to dominant mutations that may have subtle effects on the protein structure or splicing. ${ }^{20,21}$ There were no significant differences in allele frequency between cases and controls for the p.(Glu274Asp) and other synonymous variants using a $2 \times 2$ Fisher's test.

However, the substitution c.1175C $>$ T, p.(Pro392Leu) disrupts a highly conserved sequence in the UBA domain ${ }^{11}$ and may abolish the ubiquitin-binding ability of the protein. Using SIFT, Polyphen2 and Mutation taster programs, this mutation was predicted to be pathogenic by all three programs. The proband was diagnosed with ALS at the age of 53 years, and disease duration was 6 years (Figure 2a and Supplementary Information). The patient had previously been diagnosed with PDB. To confirm the presence of this mutation, we digested the PCR product with Bst $\mathrm{U} 1$, which selectively cuts the major allele leaving the mutated allele uncut (Figure 1e). We also screened for the p.(Pro392Leu) mutation in sporadic ALS cases $(n=86)$, controls $(n=78)$ and FALS cases with hexanucleotide expansions in C9ORF72 $(n=26)$. Cases with C9ORF72 expansions were included at this stage as they are known exist together with other FALS mutations. ${ }^{22}$ One further p.(Pro392Leu) mutation was detected in these FALS cases and confirmed by sequencing, but the mutation was absent from all controls and SALS cases that were screened. This FALS case showed typical ALS features with prominent bulbar features including marked tongue spasticity (Figure 2b). Disease onset occurred at 63 years of age, and disease duration was 27 months. There was no record of PDB in this kindred. Further information is available in Supplementary Information.

The overall prevalence of the p.(Pro392Leu) substitution is $2.3 \%$ in our UK-FALS cohort, which is significantly higher than the general population $(P=0.0455$, Supplementary Figure 1$)$.

c.463G $>$ A, p.(Glu155Lys) is a novel non-synonymous variant found in the ZZ-type zinc-finger domain, which is responsible for protein-protein interactions and interacts with RIP in the TNF $\alpha / N F k B$ pathway. ${ }^{11}$ The mutation is absent from all available databases and was predicted to be pathogenic by all three programs. The proband was diagnosed with ALS at 71 years and survived for 21 months and was heterozygous for the variant. There was no record of PDB in this case. The father of the proband developed PDB but not ALS, while the brother of the proband was diagnosed with ALS at 69 years. He survived for 12 months, and there was no record of PDB in this case (Figure 2c). No DNA was available for these samples.

All coding mutations in SQSTM1 detected in ALS (SALS and FALS) in this study and in previous studies are presented in Table 1 together with their prevalence in ALS and control populations and pathogenicity predictions. Analysis of all SQSTM1 variants that are predicted as being pathogenic in published cohorts, ${ }^{3-6}$ and the current study demonstrated that pathogenic SQSTM1 variants are significantly associated with FALS with a combined odds ratio of 3.91 $\left(P_{\mathrm{MH}}=0.0002\right.$, Supplementary Table 1$)$. No significant association was found for SALS cases. In combination with previous studies, it can be concluded that p.(Pro392Leu), which has been identified in five out of 517 FALS families, is the most common SQSTM1 mutation in FALS and PDB to date with a mutation frequency of $2.3 \%$ in our UK-FALS cohort and $0.97 \%$ in pooled FALS populations published to date (Table 1; Supplementary Figure 1).

We also identified two novel intronic variants, c. $205+12 \mathrm{G}>\mathrm{T}$ and c. $205+21 \mathrm{C}>\mathrm{T}$, which are located at $12 \mathrm{bps}$ and $21 \mathrm{bps}$ from the exon $1 /$ intron 1 junction, respectively. These variants were present in the same FALS individual, who was heterozygous for the first variant and homozygous for the second (Figure 1d). Their effects on splicing were analysed using the Human splicing finder program, which showed that the mutant allele of c. $205+21 \mathrm{C}>\mathrm{T}$ may give rise to a novel donor site with a consensus value of $82.06(+48.59 \%$ variation compared to wildtype) and elongate exon 1 by 16 bps.

\section{Founder haplotype of P392L SQSTM1 kindred}

A four-SNP founder haplotype, $\mathrm{H}_{2}$, has been previously described to carry most P392L mutations in PDB patients of British descent. ${ }^{23} \mathrm{We}$ carried out haplotype analysis to investigate whether this was also the case in FALS. We first reconstructed the haplotypes in the control population (1000 genome EUR group) where all four SNPs are in high LD $\left(r^{2}>0.8\right)$ (Supplementary Figure 2A), showing that $\mathrm{H}_{1}$
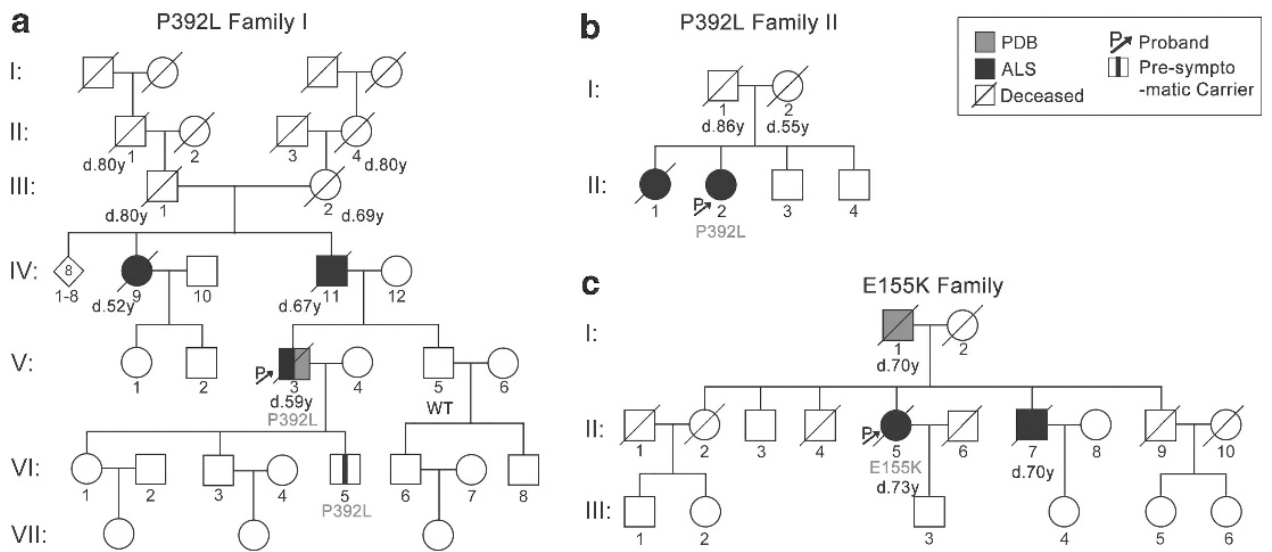

Figure 2 Pedigrees of the FALS index cases carrying p.(Pro392Leu) and p.(Glu155Lys) SQSTM1 mutations. Age of death and genotypes are indicated where available. (a) In the p.(Pro392Leu) Family I, individual II:1 and II:4 died of senility and III:1 and III:2 died of arteriosclerosis and heart disease, respectively. DNA was available for three individuals. The index case developed both PDB and ALS, and no disease phenotypes were present in generations $\mathrm{VI}$ and VII at the time of sampling. (b) In Family II, individual I:2 died of nephritis, and no DNA was available for other family members. (c) In the p.(Glu155Lys) Family, the father of the proband developed Paget's disease and died at 70 years. 
Table 1 Summary of coding variants in SQSTM1 in FALS, SALS and FTD from different studies

\begin{tabular}{|c|c|c|c|c|c|c|c|c|c|c|}
\hline \multirow[b]{2}{*}{ Protein } & \multirow[b]{2}{*}{ Position } & \multirow[b]{2}{*}{ rs } & \multirow[b]{2}{*}{ Changes } & \multirow[b]{2}{*}{ FALS } & \multicolumn{3}{|c|}{ Population prevalence } & \multicolumn{3}{|c|}{ Prediction } \\
\hline & & & & & $S A L S$ & FTD & Control & SIFT & $P P$ & $M T$ \\
\hline A33V & 179248034 & rs200396166 & c. $98 \mathrm{C}>\mathrm{T}$ & $1 / 491^{\mathrm{a}}$ & $2 / 456^{a}$ & & $6 / 4418^{a, b}$ & + & - & + \\
\hline A53T & 179248093 & & c. $157 \mathrm{G}>\mathrm{A}$ & & $1 / 456^{c}$ & & $0 / 4550^{c, b}$ & - & - & - \\
\hline M87V & 179250011 & & c. $259 A>G$ & & $1 / 456^{d}$ & & $0 / 4660^{d, b}$ & + & - & + \\
\hline K102E & 179250056 & & c. $304 A>G$ & & $1 / 456^{d}$ & & $0 / 4660^{d, b}$ & + & - & + \\
\hline V153I & 179251013 & rs145056421 & c. $457 \mathrm{G}>\mathrm{A}$ & & $2 / 456^{a}$ & & $9 / 5024^{a, b}$ & - & - & - \\
\hline E155K & 179251019 & & c. $463 \mathrm{G}>\mathrm{A}$ & $1 / 491^{\mathrm{e}}$ & & & $0 / 4300^{e, b}$ & + & + & + \\
\hline P228L & 179252155 & rs151191977 & c. $683 \mathrm{C}>\mathrm{T}$ & & $1 / 456^{a}$ & & $4 / 5024^{a, b}$ & + & - & + \\
\hline V234V & 179252174 & & c. $702 \mathrm{G}>\mathrm{A}$ & $1 / 491^{a}$ & & & $0 / 5024^{a, b}$ & - & & - \\
\hline K238E & 179252184 & rs11548633 & c. $712 A>G$ & & $1 / 456^{f}$ & & $32 / 4824^{f, b, g}$ & + & + & + \\
\hline K238del & $179252186-8$ & & c.714-716delGAA & & $1 / 456^{a}$ & & $0 / 724^{a}$ & & & + \\
\hline V259L & 179260052 & & c. $775 \mathrm{G}>\mathrm{C}$ & & & $1 / 170^{f}$ & $0 / 4445^{f, b}$ & + & - & + \\
\hline $\mathrm{H} 261 \mathrm{H}$ & 179260060 & rs145001811 & c. $783 \mathrm{C}>\mathrm{T}$ & & $1 / 456^{a}$ & & $1 / 5038^{a, b}$ & - & & - \\
\hline E274D & 179260099 & rs55793208 & c. $822 \mathrm{G}>\mathrm{C}$ & $2 / 491^{\mathrm{f}, \mathrm{e}}$ & $11 / 456^{f}$ & $5 / 170^{f}$ & $236 / 4824^{f, b, g}$ & - & - & - \\
\hline P296P & 179260165 & rs148984239 & $c .888 \mathrm{G}>\mathrm{T}$ & $1 / 491^{\mathrm{e}}$ & & & $1 / 4679^{b, g}$ & - & & - \\
\hline S318P & 179260229 & & c. $952 \mathrm{~T}>\mathrm{C}$ & $1 / 491^{a}$ & & & $0 / 5038^{a, b}$ & - & - & - \\
\hline S318S & 179260231 & rs56092424 & c. $.954 \mathrm{C}>\mathrm{T}$ & $2 / 491^{e}$ & & & $199 / 4674^{b, g}$ & - & & - \\
\hline E319K & 179260232 & rs61748794 & c955G >A & & & $1 / 170^{f}$ & $2 / 4817^{f, b, g}$ & - & - & - \\
\hline R321C & 179260238 & rs140226523 & c. $961 \mathrm{C}>\mathrm{T}$ & & $1 / 456^{a}$ & & $5 / 5407^{a, b, g}$ & + & - & - \\
\hline K344E & 179260647 & & c. $1032 A>G$ & & & $1 / 170^{f}$ & 0/4805 f,d,b & + & + & + \\
\hline V346V & 179260655 & rs150470670 & c. $1038 \mathrm{G}>\mathrm{A}$ & $1 / 491^{\mathrm{e}}$ & & & $4 / 4300^{b}$ & - & & + \\
\hline P348L & 179260660 & & c. $1044 \mathrm{C}>\mathrm{T}$ & & $1 / 456^{f}$ & & 0/4805 f,d,b & + & + & + \\
\hline S370P & 179260725 & rs143956614 & c. $1108 \mathrm{~T}>\mathrm{C}$ & $1 / 491^{a}$ & & & 0/5393a,f,b & - & - & + \\
\hline A390X & 179260783 & & c. $1165+1 G>A$ & & $1 / 456$ & & - & & & + \\
\hline P392L & 179263445 & rs104893941 & c. $1175 \mathrm{C}>\mathrm{T}$ & $5 / 517^{a, d, e}$ & $1 / 542^{a, e}$ & & $22 / 5999^{a, f, d, e, b, g}$ & + & + & + \\
\hline G411S & 179263501 & rs143511494 & c. $1231 \mathrm{G}>\mathrm{A}$ & $1 / 491^{a}$ & & & 0/5397a,d,b & + & + & + \\
\hline G425R & 179263543 & CM041449 & c. $1273 G>A$ & $1 / 491^{a}$ & & & 0/5397a,d,b & + & + & + \\
\hline P438L & 179263586 & & c. $1313 \mathrm{C}>\mathrm{T}$ & & $1 / 456^{f}$ & & $1 / 4805^{f, d, b}$ & + & + & + \\
\hline P439L & 179263676 & rs199854262 & c. $1316 \mathrm{C}>\mathrm{T}$ & & $1 / 456^{c}$ & & 0/4910 c,d,b & + & + & + \\
\hline
\end{tabular}

Mutations identified in this study are in bold, and the frequencies comprise heterozygous and homozygous minor genotypes. All changes shown are exonic except that p.Ala390*, also known as IVS7 $+1 \mathrm{G}>\mathrm{A}$, occurred at the splice site of intron 7. Data from the EUR subgroup of 1000 Genome Project and EA subgroup of NHLBI Exome sequencing project and 'in house' controls published in previous studies were combined as reference controls. The effects of mutations were predicted using SIFT, Polyphen 2 (PP) and Mutation taster (MT): '+ ' $=$ probably damaging; ' = tolerated

aFecto (FALS, $n=340$; SALS, $n=206$; control, $n=724$ )

bexome variant server, NHLBI GO exome sequencing project (ESP), Seattle, WA (URL: http://evs.gs.washington.edu/EVS/) (April, 2013).

CHirano (SALS, $n=52$; control, $n=250$ ).

Teyssou (FALS, $n=90$; SALS, $n=74$; control, $n=360$ ).

e Current study (FALS, $n=61+26$, SALS, $n=86$, control, $n=78$ ).

fRubino (SALS, $n=124$; control $=145$ ).

g1000 genomes (EUR, $n=379$ ).

(TACT) and $\mathrm{H}_{2}$ (CGTG) accounted for 95\% of haplotypes, as previously reported ${ }^{23}$ (Supplementary Table 2). All p.(Pro392Leu) mutation carriers in this study were heterozygous for $\mathrm{H}_{2}$ and $\mathrm{H}_{2 \alpha}$ (CGCG) haplotypes, and the p.(Glu155Lys) carrier was heterozygous for $\mathrm{H}_{1}$ and $\mathrm{H}_{2}$ haplotypes. In the FALS cohort as a whole, there were no significant differences in the frequency of a two-SNP haplotype between cases and controls (Supplementary Table 2). Survival data were available for 16 samples, and there were no significant differences between haplotypes (Supplementary Figure 2B).

The frequencies of common non-coding variants detected in this study are summarised in Supplementary Table 3; the allele frequencies between cases and controls were compared using $2 \times 2$ Fisher's tests and corrected for multiple testing. No marked changes were detected.

\section{DISCUSSION}

In this study, we screened all exons of the SQSTM1 gene and confirmed the presence of SQSTM1 mutations in FALS cases of UK descent. We identified six candidate exonic variants, including a novel p.(Glu155Lys) mutation, and two novel intronic variants in
FALS index cases. There were positive familial histories of Paget's disease of bone in two families carrying either the p.(Pro392Leu) or the p.(Glu155Lys) mutation, which were predicted to be pathogenic.

The p.(Glu274Asp) and other synonymous exonic variants detected in this study were not predicted to be pathogenic. In contrast to the findings of Rubino et al. ${ }^{4}$ we found no significant differences in allelic and genotypic frequencies of the p.(Glu274Asp) or other synonymous variants between our ALS cohorts and controls derived from a UK control cohort and publically available databases. In contrast, the properties of the p.(Pro392Leu) and p.(Glu155Lys) mutations are more likely to contribute pathogenic effects. First, these mutations are located in highly conserved domains that are required for the basic functions of p62/SQSTM1. The p.(Pro392Leu) mutation occurs in the UBA domain, which is required for the binding of ubiquitin, ${ }^{11}$ and modifies the structure of this domain by extending the $\mathrm{N}$-terminus of helix $1 .^{24}$ This results in a reduced affinity for mono-ubiquitin and shortened polyubiquitin chains. ${ }^{25}$ The ZZ domain, which accommodates the p.(Glu155Lys) mutation, is a conserved structural component in different ZIP/p62 homologues and has an 
important role in the formation of complexes that participate in the NFkB signalling pathway. ${ }^{26}$ Second, the coexistence of both PDB and ALS in the two families reported here and in other studies ${ }^{5}$ is indicative that common pathogenic mechanisms underlie both these diseases. P62/SQSTM1 has an established causal role in $\mathrm{PDB}$, and the p.(Pro392Leu) mutation is, in fact, the most common cause of PDB. ${ }^{7,8}$ The p.(Pro392Leu) mutation segregates with $\mathrm{PDB}$, and, although the binding of polyubiquitin remains intact, it causes a phenotype that is indistinguishable from those caused by mutations that truncate the entire domain. ${ }^{27}$ Third, multiple studies have confirmed the existence of the p.(Pro392Leu) mutation in ALS from different populations, ${ }^{3,5}$ and, in combination with the data presented here, it can be concluded that it is the most common mutation in the gene in ALS to date (Table 1). The coexistence of the p.(Pro392Leu) mutation in a family with a C9ORF72 expansion is not unexpected as C9ORF72 has also been found in FALS cases harbouring SOD1, FUS or TARDBP mutations. ${ }^{22}$ The SQSTM1 p.(Pro392Leu) mutation index case with the C9ORF72 mutation (Family II) showed classical features of ALS with no evidence of cognitive impairment and similar survival (age at death: 62-65 years) compared with Family I that lacked a C9ORF72 expansion (age at death: 52-67 years). It is possible that the severity of the disease in the proband may have been affected by the coexistence of both mutations, but whether the effect of the SQSTM1 mutation is potentiated by the expansion remains to be established.

The fundamental role of p62 in autophagy is well established and relevant to multiple tissues. However, more recently considerable evidence has accumulated for the specific involvement of p62 in ALS and ALS with fronto-temporal lobar degeneration (ALS/FTLD), where p62 is associated with both TDP-43-positive and TDP-43negative inclusions in the spinal cord and cerebral cortex. Further, similar inclusions have been demonstrated in ALS cases harbouring SQSTM1 mutations. ${ }^{5}$

In spite of the phenotypic heterogeneity found in ALS, ALS/ FTLD and PDB and related disorders such as IBMPFD and POAG, the discovery of mutations in overlapping candidate genes, such as VCP, UBQLN2, CHMP2B and OPTN, points to a common pathogenic mechanism affecting the formation and clearance of misfolded proteins. ${ }^{2}$ We previously reported that the genotypes of protein disulphide isomerase $(P 4 H B)$, a redox enzyme that reduces formation of aberrant disulphide bonds, were associated with survival time of FALS, ${ }^{28}$ supporting the hypothesis that factors interfering with proteostasis may modify disease progression and can be considered as therapeutic targets for ALS. The investigation of p62/SQSTM1 mutations in multiple cohorts provides further support for their contribution to a significant predisposition to disease in ALS.

\section{CONFLICT OF INTEREST}

The authors declare no conflict of interest.

\section{ACKNOWLEDGEMENTS}

We thank the families for their participation in this study. This study was funded by the Motor Neurone Disease Association of the UK and the Medical Research Council.
1 Robberecht W, Philips T: The changing scene of amyotrophic lateral sclerosis. Nat Rev Neurosci 2013; 14: 248-264.

2 Fecto F, Siddique T: Making connections: pathology and genetics link amyotrophic lateral sclerosis with frontotemporal lobe dementia. J Mol Neurosci 2011; 45: 663-675.

3 Fecto F, Yan J, Vemula S: SQSTM1 mutations in familial and sporadic amyotrophic lateral sclerosis. Arch Neurol 2011; 68: 1440-1446.

4 Rubino E, Rainero I, Chio A et al: SQSTM1 mutations in frontotemporal lobar degeneration and amyotrophic lateral sclerosis. Neurology 2012; 79: 1556-1562.

5 Teyssou E, Takeda T, Lebon V et al: Mutations in SQSTM1 encoding p62 in amyotrophic lateral sclerosis: genetics and neuropathology. Acta Neuropathol 2013; 1-12.

6 Hirano M, Nakamura Y, Saigoh $\mathrm{K}$ et al: Mutations in the gene encoding p62 in Japanese patients with amyotrophic lateral sclerosis. Neurology 2013; 80: 458-463.

7 Morissette J, Laurin N, Brown JP: Sequestosome 1: mutation frequencies, haplotypes, and phenotypes in familial Paget's disease of bone. J Bone Miner Res 2006; 21 P38-P44.

8 Rea SL, Walsh JP, Ward L et al: Sequestosome 1 mutations in Paget's disease of bone in Australia: prevalence, genotype/phenotype correlation, and a novel non-UBA domain mutation (P364S) associated with increased NF- $\mathrm{KB}$ signaling without loss of ubiquitin Binding. J Bone Miner Res 2009; 24: 1216-1223.

9 Keller BA, Volkening K, Droppelmann CA, Ang LC, Rademakers R, Strong MJ: Co-aggregation of RNA binding proteins in ALS spinal motor neurons: evidence of a common pathogenic mechanism. Acta Neuropathol 2012; 124: 733-747.

10 Al-Sarraj S, King A, Troakes C et al: p62 Positive, TDP-43 negative, neuronal cytoplasmic and intranuclear inclusions in the cerebellum and hippocampus define the pathology of C9orf72-linked FTLD and MND/ALS. Acta Neuropathol 2011; 122: 691-702.

11 Geetha T, Wooten MW: Structure and functional properties of the ubiquitin binding protein p62. FEBS Lett 2002; 512: 19-24.

12 Lamark T, Johansen T: Autophagy: links with the proteasome. Curr Opin Cell Biol 2010; 22: 192-198.

13 Bjørkøy G, Lamark T, Brech A et al: p62/SQSTM1 forms protein aggregates degraded by autophagy and has a protective effect on huntingtin-induced cell death. J Cell Biol 2005; 171: 603-614

14 Orrell RW, Habgood JJ, Gardiner I et al: Clinical and functional investigation of 10 missense mutations and a novel frameshift insertion mutation of the gene for copperzinc superoxide dismutase in UK families with amyotrophic lateral sclerosis. Neurology 1997; 48: 746-751.

15 Sreedharan J, Blair IP, Tripathi VB et al: TDP-43 mutations in familial and sporadic amyotrophic lateral sclerosis. Science 2008; 319: 1668-1672.

16 Vance C, Rogelj B, Hortobágyi T et al: Mutations in FUS, an RNA processing protein, cause familial amyotrophic lateral sclerosis type 6. Science 2009; 323: 1208-1211.

17 Smith BN, Newhouse S, Shatunov A et al: The C9ORF72 expansion mutation is a common cause of ALS $+1-$ FTD in Europe and has a single founder. Eur J Hum Genet 2012; 21: 102-108.

18 Chen HJ, Anagnostou G, Chai A et al: Characterization of the properties of a novel mutation in VAPB in familial amyotrophic lateral sclerosis. J Biol Chem 2010; 285: 40266-40281.

19 Mitchell J, Paul P, Chen $\mathrm{HJ}$ et al: Familial amyotrophic lateral sclerosis is associated with a mutation in D-amino acid oxidase. Proc Natl Acad Sci USA 2010; 107: 7556-7561.

20 Flanagan SE, Patch AM, Ellards S: Using SIFT and PolyPhen to predict loss-offunction and gain-of-function mutations. Genet Test Mol Biomarkers 2010; 14: 533-537.

21 Valdmanis PN, Verlaan DJ, Rouleau GA: The proportion of mutations predicted to have a deleterious effect differs between gain and loss of function genes in neurodegenerative disease. Hum Mut 2009; 30: E481-E489.

22 van Blitterswijk M, van Es MA, Hennekam EA et al: Evidence for an oligogenic basis of amyotrophic lateral sclerosis. Hum Mol Genet 2012; 21: 3776-3784.

23 Lucas GJ, Hocking LJ, Daroszewska A et al: Ubiquitin-associated domain mutations of SQSTM1 in Paget's disease of bone: evidence for a founder effect in patients of British descent. J Bone Miner Res 2005; 20: 227-231.

24 Ciani B, Layfield R, Cavey JR, Sheppard PW, Searle MS: Structure of the Ubiquitinassociated domain of p62 (SQSTM1) and implications for mutations that cause Paget's disease of bone. J Biol Chem 2003; 278: 37409-37412.

25 Cavey JR, Ralston SH, Sheppard PW et al: Loss of ubiquitin binding is a unifying mechanism by which mutations of SQSTM1 cause Paget's disease of bone. Calcified Tissue Int 2006; 78: 271-277.

26 Moscat J, Diaz-Meco MT, Wooten MW: Signal integration and diversification through the p62 scaffold protein. Trends Biomed Sci 2007; 32: 95-100.

27 Hocking LJ, Lucas GJ, Daroszewska A et al: Novel UBA domain mutations of SQSTM 1 in Paget's disease of bone: genotype phenotype correlation, functional analysis, and structural consequences. J Bone Miner Res 2004; 19: 1122-1127.

28 Kwok CT, Morris AG, Frampton J, Smith B, Shaw CE, de Belleroche J: Association studies indicate that protein disulfide isomerase is a risk factor in amyotrophic lateral sclerosis. Free Radical Biol Med 2013; 58: 81-86

Supplementary Information accompanies this paper on European Journal of Human Genetics website (http://www.nature.com/ejhg) 